\title{
NEUROMARKETING COMO HERRAMIENTA DE PLANIFICACIÓN EN LA GESTIÓN MUNICIPAL
}

\author{
NEUROMARKETING AS A PLANNING TOOL IN MUNICIPAL \\ MANAGEMENT
}

\author{
MSc. Raquel Irene Laguado Ramírez * \\ * Universidad Francisco de Paula Santander, Facultad de Ingenierías, Grupo de \\ Investigación en Innovación y Gestión Productiva GIINGPRO \\ Ave. Gran Colombia No. 12E-96 Barrio Colsag, Cúcuta, Norte de Santander, Colombia. \\ Tel: (+57) (5) 776655 Ext. 120, E-mail: raquelirenelr@ufps.edu.co.
}

\begin{abstract}
Resumen: El estudio tuvo como propósito inicial analizar el neuromarketing como herramienta de planificación en la gestión municipal. Metodológicamente basado en un paradigma post-positivista, como un estudio cualitativo etnográfico con apoyo documental, con técnicas bajo un paradigma crítico interpretativo. La información se obtuvo mediante observación de participantes y la entrevista semiestructurada a los informantes de las cuatro organizaciones municipales seleccionadas. La fiabilidad de dicha población es relativa, al igual se destinará la ética como condición imperante.
\end{abstract}

Palabras clave: Neuromarketing, Herramienta de planificación, Gestión municipal.

\begin{abstract}
The purpose of the study was to analyze neuromarketing as a planning tool in municipal management. Methodologically based on a post-positivist paradigm, as an ethnographic qualitative study with documentary support, with techniques under a critical interpretive paradigm. The information was obtained through participant observation and the semi-structured interview with the informants of the four selected municipal organizations. The reliability of this population is relative, just as ethics will be used as a prevailing condition.
\end{abstract}

Keywords: Neuromarketing, Planning tool, Municipal management.

\section{INTRODUCCIÓN}

Desde la perspectiva de que la humanidad, vive en la búsqueda constante de satisfacer sus intereses y necesidades, esta trabaja incansablemente por conseguir sus propósitos, implicando involucrase en los avances de todas las ciencias tanto sociales como científicas, lo que le permite innovar constantemente en todos los campos de acción donde se desenvuelve, lo que hace pensar que sin estas razones y deseos, se estaría en unas sociedades catatónicas, estancadas en la inexperiencia, obligadas a permanecer en ese estado por siempre.
Una vez comprendido, que los seres humanos son motivados a realizar sus objetivos y metas, de acuerdo a la jerarquización de sus necesidades, toda vez que estas han sido priorizadas, se admite por una parte el rol fundamental del marketing como una de las estrategias más utilizadas para generar, así como, descubrir necesidades en la sociedad, provocando en estas el deseo de satisfacerla; por otra parte, se concibe además como una filosofía en la que el consumo de un producto o servicio, se vuelven una necesidad requerida para completar un propósito de vida. 
Se deduce entonces, que el marketing, como puente entre el cliente y la organización, es el área que traduce los mensajes internos y externos que ambos tienen, para lo cual se enfoca en una mezcla entre el producto, el canal, el precio y la comunicación, así como en las investigaciones de mercado, para descubrir las necesidades o conocer del comportamiento del consumidor, lo que irá guiando de manera acertada a la organización para poder atender de una forma eficiente y eficaz a los clientes, permitiendo que cumpla con sus objetivos. De los planteamientos anteriores, el investigador infiere que, la aplicación o implementación del neuromarketing como técnica moderna, en el proceso de planeación para la gestión municipal resultaría funcional, en la medida que determina los servicios requeridos por los ciudadanos y estos manifiestan su satisfacción por ellos; así también aumenta el sentido de pertenencia, además de la participación ciudadana; permite un buen posicionamiento frente a los otros municipios, elevando la imagen positiva en beneficio de su renombre. De aquí la importancia de conocer, las fortalezas, así como las oportunidades que brinda, el neuromarketing dentro de las políticas de trabajo de las alcaldías, ya que pudiese generar el buen desenvolvimiento de las acciones de estos funcionarios. Además de ser esta una oportunidad para contribuir en un aporte y punto de referencia bibliográfico para el grupo de interesados de hacer del neuromarketing una herramienta fundamental en la gestión municipal. Encontrar herramientas bibliográficas en la materia, algunas veces en los estudios o investigaciones suele ser un poco complicado (Velasquez, Puentes y Espinel, 2016), de allí que la dinámica del presente estudios es un importante referente en este tema de investigación. De esta forma, un gran porcentaje de los ciudadanos, esperan que las disposiciones gubernamentales que se tomen, sea en función de resolver las necesidades que los aquejan y que los mantienen a la expectativa de los ofrecimientos, de manera que a través de los tipos de estímulos que se utilicen tomen decisiones de apoyo o rechazo.

\section{PROCEDIMIENTO TEÓRICO}

\subsection{Marketing}

Para poder comprender de manera óptima al neuromarketing, se procederá en primera instancia a definir el concepto de marketing y de neurociencia; en tal sentido (La American Marketing Association, 2011) ofrece como definición formal de marketing, a la actividad o grupo de entidades y procedimientos para crear, comunicar, entregar, e intercambiar ofertas que tienen valor para los consumidores, clientes, socios y la sociedad en general. De la misma manera, (Warren, 2009) concibe el marketing como una función organizacional y una serie de procesos para crear, comunicar y proporcionar valor a los clientes. Así mismo, las actividades se centran en las iniciativas de una organización para satisfacer los deseos y necesidades de sus clientes con productos y servicios que ofrecen valor competitivo. Por otro lado, (Armstrong, 2011) precisa que el marketing es un proceso mediante el cual la empresa crea valor para los clientes y establece relaciones sólidas con ellos, obteniendo a cambio el valor de los clientes. En este orden, plantean el proceso en cinco pasos, a saber: 1. Entender el mercado y las necesidades de los clientes, 2. Diseñar una estrategia de marketing impulsada por el cliente, 3. Elaborar un programa de marketing, 4. Crear relaciones rentables y satisfactorias para los clientes, 5 . Captar el valor de los clientes para obtener su lealtad y ventas para la empresa. Los primeros cuatro pasos, se centran en entender a los consumidores, crear valor para el cliente, y construir solidad relaciones con éste; y el último se materializa en ventas y lealtad del cliente a largo plazo. (Armstrong, 2012) plantea que el marketing es una filosofía según la cual el logro de las metas de la organización depende de conocer las necesidades y deseos de los mercados metas, así como de proporcionar las satisfacciones deseadas de mejor manera que los competidores. En este caso, el trabajo no es encontrar los clientes adecuados para el producto, sino encontrar los productos adecuados para los clientes. Así mismo, es muy importante la administración de recursos humanos, tal como es citado en (Ospina, Angulo, Suárez y Suárez, 2017) la gestión del talento humano un pilar fundamental para el desarrollo exitoso de los procesos, dado que son los gestores municipales quiénes desarrollar las actividades que conducen al éxito de la planificación municipal.

En ese mismo orden, (Kotler, 2012) refiere que el marketing trata de identificar las necesidades humanas y sociales; por lo que es satisfacer necesidades de manera rentable. En tal sentido lo define como un proceso social por el cual tanto grupos como individuos obtienen lo que necesitan y desean mediante la creación, oferta y libre intercambio de productos y servicios de valor con otros grupos e individuos. En ese 
sentido, a partir del concepto general de marketing ya es común hablar de marketing de productos frente al marketing de servicios, y más recientemente empieza a hablarse de marketing social, público y municipal. Del mismo modo, (Kotler y Lee, 2007) conciben el marketing social debe desarrollar y promover un valor superior a los consumidores, de forma que se mantenga o mejore el bienestar del consumidor y de la sociedad. Por lo tanto, los responsables de esta actividad deben incorporar las consideraciones éticas y sociales, así como también ponderar los potenciales impactos negativos de satisfacer los deseos a corto plazo del consumidor con el bienestar social a largo plazo. En ese orden de ideas, para (Alonso, 2006) el marketing público es una parte del marketing de instituciones no lucrativas circunscrita a las actividades de intercambio de las entidades públicas, especialmente los servicios públicos que presta la Administración del Estado. El marketing público ha sido definido por (Chias, 2007) como la ciencia de los procesos de intercambio públicos y tiene por objetivo la satisfacción de las necesidades públicas, de forma eficiente y siempre a partir de los recursos disponibles. Para este autor la eficacia, la eficiencia y la legalidad presupuestaria serían los elementos clave de una buena gestión de marketing público.

De allí que también, (Stokes y Lomax, 2008) comentan que el marketing público tiene necesariamente que ganar protagonismo en la gestión pública en los próximos tiempos a fin de satisfacer las necesidades de los ciudadanos de forma eficaz y efectiva. En definitiva, lo que busca es tratar de lograr una administración al servicio de la sociedad como tal liderizando y obviando la anterior en cuanto a administración de control se refiere, desarrollando para ello una actitud pro activa de servicio a los ciudadanos, facilitando la consecución de los objetivos que se citan como lugar común de esta nueva perspectiva, a saber: el aseguramiento de la convivencia, el bienestar; las garantías de los derechos de los ciudadanos frente a actos arbitrarios no justificados, mejorar sus relaciones con la ciudadanía.

\subsection{Neurociencia}

La neurociencia en palabras de (Casafont, 2012) señala que la complejidad que supone la investigación del cerebro trae consigo que la neurociencia utilice el enfoque reduccionista, con el que se pretende hacer un estudio de las partes, en lugar de uno del todo. Para ello se han descrito niveles de complejidad creciente, desde el estudio de las moléculas implicadas en nuestra estructura cerebral, siendo este el más elemental, hasta el estudio de las funciones cognitivas, de connotación más compleja; y entre los mismos niveles, el estudio de células de los sistemas, como también de la conducta.

En este sentido, (Manes, 2014) indica que la neurociencia estudia la organización y el funcionamiento del sistema nervioso, así como también, los diferentes elementos del cerebro que interactúan y dan origen a la conducta de los seres humanos. En ese orden, revisa los fundamentos de la individualidad, tales como las emociones, conciencia, toma de decisiones y las acciones sociopsicológicas. Por otra parte, como todo se hace con el cerebro, es lógico que el impacto de la neurociencia se proyecte en múltiples áreas de relevancia social. Por tanto, al tratarse de un área tan importante para el conocimiento humano, resulta comprensible y necesario que los procesos de las neurociencias no queden solamente en los laboratorios, sino que sean absorbidos y debatidos por la sociedad en general.

De la misma manera, el autor en mención explica que la neurociencia ha realizado aportes considerables para el reconocimiento de las intenciones de los demás y de los distintos componentes de la empatía, de las áreas críticas del lenguaje, mecanismos centrales de la emoción, y de la misma manera de circuitos neutrales involucrados en ver e interpretar el mundo que rodea a la sociedad. Es así como la neurociencia y los sistemas neuronales permiten una interacción entre los seres humanos o grandes gestores para lograr una efectiva planificación de lo proyectado, (Camargo, Coronel, y Calderon, 2015) presentan una investigación donde una serie de redes neuronales le permite al ser humano interactuar con la máquina para lograr un hogar inteligente que facilita su vida cotidiana.

Así mismo, investigaciones en torno a los sistemas de redes neuronales (Gualdrón, Durán, Araque, y Ortíz, 2014; Vides, Pardo, y Torres, 2014) demuestran que este ámbito de estudio proyecta un sistema de planificación conducente al logro de objetivos y gestión que facilita el desarrollo de la sociedad y en la solución de problemas (Contreras, Florez y Cortes, 2012; Florez, Peña, y Laguado, 2015) 


\subsection{Neuromarketing como herramienta de planificación en la gestión municipal}

En la actualidad, uno de los mayores retos de la gestión moderna de empresas es indagar la forma en que interactúan los procesos cognitivos racionales e irracionales en los intereses y toma de decisiones de las personas. Las neurociencias buscan entender los procesos a nivel celular y molecular por los cuales el cerebro produce actos de comportamiento y cognitivos específicos, y es precisamente la investigación y comprensión de todo lo que acontece en el cerebro humano y determina el comportamiento de las personas lo que hará más exitosas las estrategias dirigidas de las organizaciones modernas.

Como en todas las ramas de la administración y gracias a la importancia que tiene el marketing hoy en día, importantes pensadores, expertos en múltiples ciencias, empíricos y teóricos, crean e inventan nuevas técnicas y tecnologías para mejorar, fortalecer o hacer más confiables y rentables a las organizaciones, para así mantenerla sostenible y perdurable. En ese sentido esta área de gestión no es ajena a esto y dentro de las muchas cosas que se han propuesto para aplicar dentro de este campo se encuentra en auge el Neuromarketing; que entre otras cosas se la han dado muchas definiciones en los últimos años.

Para (Solomón, 2008) el comportamiento del consumidor conocido como neuromarketing es la interacción dinámica de los efectos, cognición, comportamiento y ambiente, mediante las cuales los seres humanos llevan a cabo los aspectos de intercambio comercial de su vida, en otras palabras, el comportamiento del consumidor abarca pensamientos y sentimientos que experimentan las personas, así como las acciones que emprenden, en los procesos de consumo.

También (Braidot, 2009) señala que el Neuromarketing es una disciplina de avanzada, que tiene como función investigar y estudiar procesos cerebrales que hacen de una manera más clara la conducta y toma de decisiones de las personas en los campos de acción de marketing tradicional (inteligencia de mercado, diseño de productos y servicios, comunicaciones, precios, posicionamiento, targeting, canales, ventas, fidelización).

En tal sentido, dice que se puede examinar la mente del consumidor, conocer sus deseos, que es lo que los motiva para hacer, a su parecer, la mejor toma de decisión a la hora comprar o consumir un producto o servicio independientemente del tamaño de la organización con la que se esté trabajando, el producto que se quiera vender o al tipo de consumidor a los cuales se quieran dirigir. También incluye todo lo relativo al ambiente que influye en esos pensamientos, sentimientos y acciones, ello complementará comentarios de otros consumidores, anuncios, información de precio, empaques, aspectos del producto y otros factores más.

Plantea el mismo autor, que, al aplicar nuevas metodologías de investigación, junto a los conocimientos que se están generando en la neuropsicología, las neurociencias y la antropología sensorial, el neuromarketing facilita la comprensión de las verdaderas necesidades de los clientes y permite superar potenciales errores por desconocimiento de sus procesos internos y meta consciente. De la misma manera dice que con un diseño adecuado, el neuromarketing puede informar qué está pasando en el cerebro de un cliente ante los diferentes estímulos que recibe, brindando un campo de estudios mucho más potente que el que suministró el marketing tradicional debido a sus limitaciones para explorar los mecanismos meta consciente.

De igual modo, explica que esta nueva técnica trae consigo beneficios tales como: capacidad para planificar estratégicamente y diseñar acciones sorpresa para los clientes; diseños de productos o servicios vinculados con las percepciones que inciden posteriormente en las decisiones de compras de los clientes; habilidades para generar una mejor relación con las personas, entre otras. Por otra parte, (Hassan, 2009) tiene planteado en su concepción que el neuromarketing es un curioso matrimonio entre el marketing y la ciencia, es la llave que se denomina la lógica de comprar: los pensamientos, sentimientos y deseos subconscientes que mueven las decisiones de compra que tomamos todos los días de nuestras vidas. Así también (Olamendi, 2008) dice que hoy en día las técnicas que utiliza la neurociencia son de índole psicofísica con constante uso de imágenes de resonadores magnética, magnetoencefalógrafo y electroencefalógrafos, en el entendido que la neurociencia detectó la dificultad e imposibilidad por parte de los consumidores de expresar las razones emocionales que generan sus hábitos de consumo, y sus reacciones a los distintos estímulos del mercado. Plantea también el autor al referirse al neuromarketing, que el conocer el funcionamiento 
del cerebro en las decisiones acerca del producto, o, dicho de otra manera, de cómo las personas eligen, es un nuevo campo del marketing que permite investigar la respuesta cerebral a los estímulos publicitarios, de marca y de otros tipos de mensajes culturales. Todo lo anterior, para decir que el neuromarketing se refiere al estudio del funcionamiento del complejo proceso de comunicación entre el ser humano vendedor y el ser humano cliente; y en ese orden de ideas, se ocupa de analizar las sensaciones que experimenta el consumidor durante el proceso de compra de un producto o servicio.

De igual manera (Cisneros, 2012) plantea que el neuromarketing es igual al marketing emocional; y concibe emoción como un sentimiento producido por el subconsciente ante la oportunidad de satisfacer una necesidad básica que impulsa al cuerpo a actuar para conseguirla; por lo que el ser humano es un ser emocional dominado por sus sentimientos y emociones, antes que por sus razones; dado a que una mente emocional es mucho más rápida que la mente racional. También afirma que los sentimientos influyen en el funcionamiento del cerebro y son básicos a la hora de tomar decisiones. Por otra parte (Klaric, 2012), considerado como el gurú del neuromarketing y experto número uno en neuroinnovación; dice que en los últimos diez años se ha generado más conocimiento de cómo opera la mente humana y como se fundamenta el comportamiento humano; probándose que más del $85 \%$ de la decisión de todo lo que se hace en la vida, la compra de un producto o servicio, donde vas a vivir, o que sientes por una persona, proviene del subconsciente.

En tal sentido, concibe el neuromarketing como una herramienta muy importante en descifrar los patrones de conducta neurológica; el cual permite saber claramente si el cerebro se deja seducir o no por ciertos estímulos. De la misma manera plantea el autor que a través de él se busca el conocimiento necesario para alinear a los distintos agentes de activación y comunicación. Pero que sin embargo, se hace necesario el uso mixto (herramientas de las ciencias sociales junto con las de ciencia científica).

\section{PROPÓSITO Y METODOLOGÍA}

La ruta metodológica que se implementa para el avance del estudio considera desde sus planteamientos los distintos elementos que involucran el contexto de la investigación, aunado a la información, quien es la que determina la técnica que se emplea para el análisis y los procedimientos que se utilizan para dilucidar los resultados. De manera que, se puede contextualizar el conocimiento de forma explícita, para lo cual, es importante considerar la epistemología, dando respuesta a las interrogantes formuladas para lograr obtener hallazgos significativos.

Desde esta perspectiva, la investigación tiene un enfoque cualitativo post positivista, que de acuerdo con (Martínez, 2006), este paradigma conlleva el trabajo sobre el campo subjetivo donde el enlace sujeto-objeto tiene una connotación más hacia la interpretación que hacia la medición, donde el valor es adjudicado a la situación que se aborda, que al número que esta representa, por ello, este paradigma se convierte en la plataforma doctrinaria epistémica para el desarrollo de este estudio.

Siguiendo el punto de vista cualitativo, la investigación se alude al paradigma en un sentido epistemológico como marco de referencia para la investigación científica, llámese esta cuantitativa o cualitativa siendo esta ultima la que se desarrolla en la presente investigación. En tal sentido, partiendo de la orientación epistemológica que para la presente investigación se toman como fundamentos filosóficos los que plantean (Habermas, 2008) es preciso anotar lo siguiente: Por una parte, las teorías, perspectivas creadoras de imágenes del objeto, método y sujeto del conocimiento, se conciben como partes del paradigma que las engloba. Los paradigmas deben entenderse como sistemas de creencias básicas sobre: a) La naturaleza de la realidad investigada, supuesto ontológico. La identificación de un paradigma se produce al conocer cuál es la concepción o creencia que sostiene la investigadora o el investigador respecto a la naturaleza de la realidad investigada. b) El modelo de relación entre la investigadora o el investigador y lo investigado, supuesto epistemológico. c) El modo en que podemos obtener conocimiento de dicha realidad, supuesto metodológico. Tiene que ver con los métodos y procedimientos que se derivan de las posturas adoptadas en los niveles ontológico y epistemológico.

En el mismo sentido, dentro de las metodologías cualitativas, el enfoque etnográfico es, quizás, uno de los de más vieja data. En tanto, en muchas de las historias que narra, describe e interpreta las realidades observadas desde el punto de vista conceptual de sus protagonistas. Por ello, el 
investigador etnográfico, al desear acercarse a la verdadera naturaleza de las realidades humanas, se centra en la descripción y la comprensión. Por lo que quiere profundiza en su investigación con una mente lo más abierta posible, permitiendo que vayan emergiendo las impresiones con sus relaciones.

La etnografía según (Giddens, 2010), es visto como el estudio directo de personas o grupos durante un cierto período, utilizando la observación participante o las entrevistas para conocer su comportamiento social. Mientras que, Rodríguez citados por (Barbolla, et al., 2010), la define como el método de investigación por el que se aprende el modo de vida de una unidad social concreta, pudiendo ser ésta una familia, una clase, un claustro de profesores o una escuela.

Se deduce de todo lo anterior, que la etnografía es un proceso sistemático de aproximación a una situación social, considerada de manera global en su propio contexto natural; siendo su principal objetivo la comprensión empática del fenómeno objeto de estudio, de allí que se interesa por lo que la gente hace, cómo se comporta, como interactúa; proponiéndose a descubrir sus creencias, valores, motivaciones entre otros aspectos.

Por lo anteriormente expuesto, cabe anotar que esta investigación, según lo planteado por el autor antes referido, es apuntalada en el postulado de que las creencias, roles, valores y normas del ambiente en los cuales se vive, se interiorizan de manera paulatina para hacer surgir peculiaridades que explican la conducta tanto individual como grupal, de manera sistematizadamente adecuada, en el entendido de la manifestación de diferentes aspectos de la vida, compartiendo una estructura lógica de razonamiento.

Cabe destacar, que en esta investigación se prevé desarrollar una comunicación directa con los informantes claves, porque el propósito del estudio es analizar el neuromarketing como herramienta de planificación en la gestión municipal, a partir de su análisis por la experiencia de los alcaldes, secretarios de despacho, propuestas de aspirantes a alcaldías, exsecretarios y usuarios líderes, utilizándose para ello la observación directa del investigador en su vivencia como parte de una campaña y la entrevista como técnica para la recolección de datos. Entendiendo la observación como factor fundamental para determinar el diseño de la investigación, se plantea que según (Tamayo y Tamayo, 2012) este es la estrategia que el investigador adopta para poder dar respuesta al fenómeno que se estudia, es decir, como se concibe y obtiene la información, provocando direccionar al investigador sobre lo que debe hacer para alcanzar sus propósitos, obteniendo respuestas a las interrogantes que surgen a razón del estudio.

Específicamente para esta investigación, se presenta un diseño bibliográfico descriptivo, sobre el cual (Méndez, 2006) aporta que es un proceso que se basa en la exploración, análisis, desempeño, interpretación y diatriba de datos recolectados principalmente, de libros, revistas, periódico, es decir, de las fuentes primarias y secundarias, en este sentido, la primera se fundamenta en testimonios de personas (informantes clave), que han vivido al suceso que aborda la investigación, los cuales quedarán registrados como documentos para futuras investigaciones, la segunda son los obtenidos y registrados por otros investigadores en fuentes impresas, documentales, audiovisuales o electrónicas.

En este sentido, considerando el enfoque de la investigación, como cualitativo de tipo fenomenológico etnográfico, es preciso evaluar desde el diseño lo que señala (Salgado, 2007), refiriendo el diseño como un abordaje global donde se emplea el proceso de investigación, flexible y abierto, así como el desarrollo de esta se rige por el campo, ajustándose así a las condiciones del escenario o contexto.

\section{CONCLUSIONES}

Buscando finalmente dar cumplimiento a un propósito más específico en cuanto a orientaciones teóricas sobre filosofía organizacional, con base a las experiencias obtenidas de los actores involucrados, cuyas capacidades, habilidades y destrezas los hacen valiosos para la empresa. Gracias al proceso de triangulación, se desprenden los aciertos significativos que surgen de la investigación, el cual, pasando por toda la revisión bibliográfica, llega al análisis e interpretación de la información, para poder proponer los aciertos que a continuación se presentan:

Es una realidad que las Alcaldías, han logrado desempeñarse exitosamente en dos aspectos relevantes; en primera instancia, destacan que una de las mejores formas de incrementar la efectividad, es realizando las acciones de manera distinta a la forma tradicional, incorporando de esta manera el cambio como una constante en 
fortalecimiento de su gestión; y por la otra parte, el hincapié puesto en la investigación considerando los nuevos enfoques, así como las teorías gerenciales para adecuarlos a su funcionamiento.

Sin embargo, dados los avances que dentro de la gestión municipal se puedan considerar, existe un conocimiento débil de los aportes que el neuromarketing, pudiese estar brindando como herramienta de planificación. Para esto, se examinaron las técnicas aplicadas por el neuromarketing como herramienta de planificación de la gestión municipal, arrojando como resultado dentro del análisis comparativo, un generalizado desconocimiento de técnicas, que deben considerarse al momento de emprender un plan de desarrollo estratégico en un contexto de cambio.

En este sentido, la complejidad del actual entorno social muchas veces satura la capacidad de gestión, debido a la cantidad de necesidades sociales, donde ciudadanos demanda mayor atención, por estar experimentando un creciente proceso de globalización, lo que hace cada vez más difícil ser competitivo, se necesita dinamismo, capacitación y acción orientada hacia el logro de los objetivos.

Se precisó que los miembros de las municipalidades; pueden darse cuenta de las necesidades de la población con tal sólo observar su manera de vestir, o escuchar su forma de referirse, sin embargo, muchas veces se hace caso omiso a estas carencias; en tanto, la orientación de los empleados está más enfocada en el desarrollo laboral, que en las verdaderas necesidades, que en sí, son las que generan satisfacción de acuerdo a los resultados que se obtengan luego del desempeño en la gestión pública.

\section{REFERENCIAS}

Alonso, J. (2013). Comportamiento del consumidor (8va ED). ESIC EDITORIAL. Madrid, España.

American Marketing Association, Committee on terms. (2011). Marketing Definitions: A Glossary of Marketing Terms. Chicago, R.S. Alexander (Chairman).

Armstrong, G. (2011). Principles of Marketing. 14th Edition. Trang Pham

Armstrong, G. (2012). Principles of Marketing. 15th Edition. Trang Pham

Barbolla, C. Benavente, Martínez N; López T; Almagro, C., y Serrano, C. (2010). Investigación etnográfica. Métodos de Investigación Educativa en Ed. Especial. 3o Ed.
Especial. Disponible en: http://www.uam.es/personal_pdi/stmaria/jmurill o/InvestigacionEE/Presentaciones/Curso_10/I_ Etnografica_Trabajo.pdf.

Braidot, N. (2009) Neuromarketing: ¿Por qué tus clientes se acuestan con otro si deciden que les gustas tú?, Editorial Arte S.A. Venezuela.

Camargo, E. Coronel, C y Calderon, M. (2015) Hogar inteligente por control de voz usando redes neuronales. Revista Colombiana de Tecnologías de Avanzada. Vol. 1, 25, pp 16-20.

Casafont, R. (2012). Viaje a tu cerebro. Primera Edición; Editorial Ediciones B, S.A. Barcelona España.

Chias, J. (2007). El marketing público, McGraw Hill, Aravaca.

Cisneros, E. (2012). Neuromarketing y Neuroeconomía. Segunda Edición, Editorial Ecoe Ediciones Bogotá Colombia.

Contreras, G., Flórez, E. y Peña, C. (2012) Aplicación social de la asignatura diseño mecánico en la carrera ingeniería mecánica. Revista Colombiana de Tecnologías de Avanzada. Vol. 2, 20, pp 65-71.

Florez, E., Peña, C. y Laguado, R. (2015) Aplicación del método de la ecuación de Boltzmann en redes para la simulación bidimensional de un problema típico de mecánica de fluidos. Revista Colombiana de Tecnologías de Avanzada. Vol. 1, 25, pp 119125.

Giddens, A. (2010). The consequences of modernity. Stanford, CA: Stanford University Press.

Gualdrón, O., Durán, C., Araque, J. y Ortíz, J. (2014) Implementación de un modelo neuronal en un dispositivo hardware (FPGA) para la clasificación de compuestos químicos en un sistema multisensorial (nariz electrónica) Revista Colombiana de Tecnologías de Avanzada. Vol. 2, 24, pp 127-133.

Habermas, J. (2008). Sobre el concepto de opinión pública. En: Historia y crítica de la opinión pública. Barcelona: Gustavo Gilli, pp. 261-274.

Hassan, Y. (2009). La experiencia del usuario. Retrieved, Retrieved from http://www.nosolousabilidad.com/articulos/exp eriencia_del_usuario.htm

Klaric, J. (2012) Estamos Ciegos. Primera Edición, Editorial Planeta Colombiana S.A. Bogotá Colombia.

Kotler, P. (2012). Marketing 3.0. Segunda Edición, Editorial Ediciones de la U, Bogotá Colombia.

Kotler, P. y Lee, N. (2007). Marketing para el sector público; México, Editorial McGraw Hill. 
Manes, F. (2014), ¿Cómo usar el 100\% del cerebro?. Publicado en el Diario El País (Colombia) por F.M.

Martínez, M. (2006). La Investigación Cualitativa Etnográfica. Tercera Edición, Editorial Trillas México.

Méndez, C. (2006) Guía para elaborar diseños de investigación en Ciencias Económicas, Contables y Administrativas Mac Graw Hill.

Olamendi, G (2008) Neuromarketing: Las Neurociencias aplicadas al Marketing. Disponible en: https://www.emprendices.co/neuromarketinglas-neurociencias-aplicadas-al-marketing/.

Ospina, M., Ángulo, J. Suárez, A. y Suárez, S. (2017) Mejora del sistema de alimentación en las máquinas multiplack y rovema para reducir el riesgo ergonómico y los tiempos improductivos de operación en las empresas manufactureras de chocolate. Revista Colombiana de Tecnologías de Avanzada. Vol. 1, 29, pp 1-8.

Salgado, A. (2007) Investigaciones cualitativa: Diseños evaluación del rigor metodológico y retos. Universidad de San Martin de Porres. Lima, Perú.
Solomón, M. (2008) Comportamiento del consumidor Séptima edición. Michael Solomón.

Stokes, D. y Lomax, W. (2008). Marketing: A Brief Introduction, Thomson Learning.

Tamayo y Tamayo, M. (2012). El Proceso de la Investigación Científica. $5^{\circ}$ edición. Venezuela: Limusa.

Velasquez, T., Puentes, A. y Espinel, E. (2016) Marco ontológico para la estructuración semántica y la recuperación de recursos bibliográficos empleando procesamiento del lenguaje natural. Revista Colombiana de Tecnologías de Avanzada. Vol.2, 28, pp 9-15.

Vides, C., Pardo, A. y Torres, I. (2014) Módulo robótico de seguimiento solar usando MPPT supervisado y controlado vía ethernet. Revista Colombiana de Tecnologías de Avanzada. Vol. 1, 23, pp 112-119.

Warren, J. (2009). Marketing internacional. Edición: 5a ed. Naucalpan de Juárez: Pearson. 